\title{
A CASE OF CUTANEOUS LEISHMANIASIS?
}

\author{
A Whitton, General Practitioner \\ Lancaster
}

Andrew Whitton has recently been serving in the $R A M C(V)$ in Iraq. Readers may find the following case of some interest. Certainly it provided a clinical dilemma of both diagnosis and management.

A young soldier belonging to the 9/12th Lancers attended the medical centre at Abu Naji camp on 19th November 2003. He had recently been on surveillance operations along the Iraq-Iran border southeast of Baghdad, monitoring the illicit but profitable smuggling routes between the two countries. Part of the area is the marshland that was forcibly drained by Saddam Hussein in response to the failed rebellion against his regime in 1991 by the Marsh Arabs.

The soldier had been in theatre for over a month. He presented with three dry crusted cutaneous lesions over his trunk. The lesions were painless without any regional lymphadenopathy. He was systemically well without signs of further infection particularly affecting the muco-cutaneous junctions of his nose or mouth.

Management depended on establishing an exact diagnosis. This presented us with problems as the nearest military hospital is a four-hour road journey away to the south of Basra. Eventually a decision was made to evacuate him by helicopter to the British Military Hospital at Shaibah where he was subsequently admitted. A biopsy was not undertaken as the laboratory lacks the specialised tests required to confirm the clinical diagnosis. He was treated with systemic flucloxacillin for five days and after an uneventful recovery discharged back to his unit in theatre.

\section{DISCUSSION}

Cutaneous Leishmaniasis (CL) is a parasitic infection caused by the protozoa Leishmania. CL is characterised by the development of single or multiple cutaneous papules on the site of a sandfly bite. These often evolve into nodules and ulcers which although healing spontaneously leave a depressed scar.

It is estimated that 12 million people worldwide are infected, with 400,000 new cases annually. Interestingly the lesion is known colloquially as the Baghdad/Delhi boil, presumably relating to its frequent occurrence in previous generations of the British Army. It is found throughout the Middle East.
The incubation period is usually a shorter time in visitors to endemic areas than locals. Lesions appear between two and four weeks after the bite.

Symptoms are noduloulcerative lesions, usually asymptomatic, which may become painful if secondarily bacterially infected. The number of lesions depends on the circumstances of the exposure and the extent of the infection with the sandfly.

Only direct diagnosis is completely reliable. Material is obtained by biopsy for staining and specialised microscopy techniques. A variety of serological test exists detecting antibody status after CL has been present for some weeks.

Most forms of $\mathrm{CL}$ are self-limiting and require no treatment other than protection from secondary infection. When further treatment is needed systemic pentavelent antimony is usually effective.

\section{CONCLUSION}

This case illustrates a possible case of CL which remains unproven. The diagnosis was made on the clinical picture, but unfortunately proved impossible to substantiate histologically.

The subject of unusual infections is particularly topical in primary care where physicians are seeing more signs and symptoms of diseases not normally encountered in the United Kingdom. This is the result of greater availability of travel to exotic destinations and the deployment of the armed services to wider operational bases.

As physicians we must maintain a high index of clinical suspicion, particularly with regard to travellers.

\section{FURTHER READING}

WHO (1990) Control of Leishmaniasis. Technical Report series 793. World Health Organisation, Geneva

Color Atlas and Symptoms of Clinical Dermatology 2001, 4th Edition. Fitzpatrick et al 